A paraître dans Revue Tiers Monde, Nº188, Décembre 2006, pp. 773-798

\title{
Conditions, résultats et perspectives d'utilisation du coton génétiquement modifié (coton Bt) dans les PED
}

\author{
Michel FOK (CIRAD, France)
}

\section{Introduction}

La commercialisation à grande échelle des variétés génétiquement modifiées, à partir de la campagne 1996/97, a exacerbé le débat [1] sur la pertinence de ces variétés pour les pays en développement (PED). D'un côté, on les voyait comme la solution pour permettre aux PED de rattraper leur retard de productivité et de résoudre le problème de la faim dans le monde [2, 3]. De l'autre, les critiques ou suspicions étaient de plusieurs ordres. On relevait l'inadéquation des variétés disponibles pour contribuer au problème de la faim [4]. On soulignait les risques de soumettre les paysans pauvres au pouvoir de monopole des semenciers [5] en leur faisant adopter une solution technique dont on mettait en doute l'efficacité. On alertait sur les risques environnementaux, liés à l'évolution de la faune ou de la flore en conséquence de l'utilisation des variétés génétiquement modifiées [6]. Sur ce plan, on a plus particulièrement mis en avant la crainte de l'émergence de ravageurs résistants aux toxines Bt produites dans les plantes génétiquement modifiées [7]. Le débat est devenu si passionné, voire irrationnelle parfois, que d'aucuns appellent à davantage de sérénité et de méthode pour cerner l'utilisation des OGM en agriculture [8, 9], en invitant à fonder les analyses sur les études de cas [10].

La commercialisation à grande échelle de cotonnier génétiquement modifié (CGM) dans les pays en développement (PED), ou considérés comme tels (l'Afrique du Sud et la Chine dès 1997/98, et l'Inde en 2002), a concerné essentiellement les variétés résistantes à certains ravageurs incorporant un gène $\mathrm{Bt}$ (coton $\mathrm{Bt}$ ). Après dix ans d'utilisation, le recul permet d'apprécier les résultats obtenus.

Le CGM n'est cependant pas une nouveauté technologique habituelle. Son développement a nécessité des investissements privés très élevés. Sa diffusion est accompagnée de règles nouvelles et particulières pour son utilisation. Ce sont ces règles qui déterminent pour beaucoup les impacts, notamment économiques, de leur utilisation. C'est une certaine variation de ces règles entre les pays utilisateurs, méconnue ou insuffisamment prise en compte, qui peut expliquer la diversité des impacts obtenus. L'objet de cet article est d'évaluer ces impacts en relation avec les conditions de diffusion. Cette approche devrait permettre de mieux appréhender les perspectives d'utilisation du CGM dans les PED.

La deuxième partie est centrée sur l'analyse des innovations institutionnelles qui ont accompagné la diffusion du CGM dans le monde. La troisième partie propose une synthèse des résultats d'utilisation du CGM pour souligner les différences suivant les modes de production et les niveaux économiques des pays. La quatrième partie est une analyse rétrospective des effets anticipés, positifs ou négatifs, au regard des résultats aujourd'hui disponibles. La conclusion précise les conditions, en particulier économiques et organisationnelles, pour que l'utilisation soit réellement bénéfique aux PED.

\section{Analyse des conditions d'utilisation du CGM}

On recense aujourd'hui près de 90 millions d'ha de cultures génétiquement modifiées. On recense environ 10 millions d'hectare de CGM, essentiellement dans sept pays (USA, Chine, Inde, Australie, Argentine, Mexique, Afrique du Sud). La diffusion a subi peu de retard dans les PED où c'est l'utilisation du CGM prédomine (Tableau 1), l'utilisation de cultures vivrières génétiquement modifiées (maïs, soja, colza) y est le plus souvent proscrite.

La diffusion de cultures génétiquement modifiées a été lancée avec la mise en œuvre de conditions ou de règles nouvelles qui imposent des engagements spécifiques aux utilisateurs concernés. Ces règles correspondent à de véritables innovations institutionnelles, c'est-à-dire des changements par rapport aux règles qui préexistaient dans le domaine de l'utilisation des semences. Lorsque ces innovations sont formalisées et transcrites dans des contrats très 
explicites, l'on peut parler d'innovation contractuelle. Il y a cependant une certaine diversité dans les conditions d'utilisation du CGM entre les pays concernés. Compte tenu de l'incidence de ces conditions sur les impacts d'utilisation du CGM, il est important de restituer la diversité et l'évolution en cours de ces conditions.

Tableau 1. Evolution des superficies de cultures d'OGM dans le monde

\begin{tabular}{|c|c|c|c|c|c|c|c|c|c|c|}
\hline & \multicolumn{9}{|c|}{ Toutes cultures, millions d'ha } & \multirow[b]{2}{*}{2005} \\
\hline & 1996 & 1997 & 1998 & 1999 & 2000 & 2001 & 2002 & 2003 & 2004 & \\
\hline USA & 1,50 & 8,10 & 20,50 & 28,70 & 30,30 & 35,70 & 39,00 & 42,80 & 47,60 & 49,80 \\
\hline Canada & 0,10 & 1,30 & 2,80 & 4,00 & 3,00 & 3,20 & 3,50 & 4,40 & 5,40 & 5,80 \\
\hline Australie & & 0,10 & 0,10 & 0,10 & 0,20 & 0,20 & 0,10 & 0,10 & 0,20 & 0,30 \\
\hline Chine & & & & 0,30 & 0,50 & 1,50 & 2,10 & 2,80 & 3,70 & 3,30 \\
\hline Mexique & & & & & & & & & 0,10 & 0,10 \\
\hline Argentine & 0,10 & 1,40 & 4,30 & 6,70 & 10,00 & 11,80 & 13,50 & 13,90 & 16,20 & 17,10 \\
\hline Brésil & & & & & & & & 3,00 & 4,00 & 9,40 \\
\hline Af. Du Sud & & & & 0,10 & 0,20 & 0,20 & 0,30 & 0,40 & 0,50 & 0,50 \\
\hline Inde & & & & & & & & 0,10 & 0,50 & 1,30 \\
\hline Autres & 0,00 & 0,10 & 0,10 & 0,00 & 0,00 & 0,00 & 0,20 & 0,20 & 2,80 & 2,40 \\
\hline \multirow[t]{3}{*}{ Total } & 1,70 & 11,00 & 27,80 & 39,90 & 44,20 & 52,60 & 58,70 & 67,70 & 81,00 & 90,00 \\
\hline & \multicolumn{9}{|c|}{ Coton, million d'ha } & \\
\hline & 1996 & 1997 & 1998 & 1999 & 2000 & 2001 & 2002 & 2003 & 2004 & 2005 \\
\hline USA & 0,80 & 1,40 & 2,30 & 3,20 & 4,60 & 5,00 & 4,50 & 4,20 & 4,30 & \\
\hline Australie & & & 0,08 & 0,12 & 0,12 & 0,14 & 0,07 & 0,09 & 0,25 & \\
\hline Chine & & & 0,06 & 0,30 & 0,50 & 1,50 & 2,10 & 2,80 & 3,70 & \\
\hline Mexique & & & 0,04 & 0,02 & 0,02 & 0,02 & 0,02 & 0,03 & & \\
\hline Argentine & & & & 0,01 & 0,02 & 0,02 & 0,02 & 0,02 & & \\
\hline Inde & & & & & & & 0,04 & 0,10 & 0,05 & \\
\hline Autres & & & & 0,01 & 0,03 & 0,06 & 0,05 & 0,03 & 0,70 & \\
\hline Total & 0,80 & 1,40 & 2,48 & 3,66 & 5,29 & 6,74 & 6,80 & 7,27 & 9,00 & 9,80 \\
\hline$\%$ Surf. mond & $2 \%$ & $4 \%$ & $8 \%$ & $11 \%$ & $17 \%$ & $20 \%$ & $23 \%$ & $23 \%$ & $25 \%$ & $27 \%$ \\
\hline
\end{tabular}

\section{II.1. Innovation contractuelle avec une certaine diversité d'application}

Depuis son commencement, en 1996, la commercialisation du CGM s'est accompagnée d'une innovation institutionnelle, due à la multinationale Monsanto, appliquée d'abord aux EtatsUnis avant d'être étendue à d'autres pays.

L'innovation institutionnelle a consisté à obliger le paysan utilisateur à signer un contrat par lequel il accepte le paiement d'une redevance d'emploi et l'interdiction de l'utilisation de la production obtenue comme semences paysannes ${ }^{1}$. Dans le cas du coton $\mathrm{Bt}$, il doit aussi s'engager à installer une parcelle dite de refuge, semée avec des semences conventionnelles.

Par cette démarche contractuelle, le prix des semences n'est pas réellement différent de celui des semences de coton conventionnel mais la valeur de la redevance s'y ajoute. Ce système s'est cependant appliqué à des pays où les semences étaient déjà cédées au prix de marché, de sorte que la redevance représentait le seul surcoût. Ce n'est pas le cas dans tous les PED, comme les pays de l'Afrique Zone Franc, et cette différence est importante à considérer [11].

La redevance d'emploi est jugée généralement élevée ${ }^{2}$, même par les utilisateurs de pays industrialisés. Il n'est pas aisé de reconstituer les valeurs de la redevance d'emploi des semences de coton Bt dans les différents pays. Nous en proposons une première synthèse, qui reste imparfaite, en montrant aussi le différentiel du prix des semences dans les pays où la redevance n'est pas appliquée (Tableau 2).

\footnotetext{
${ }^{1}$ C'est-à-dire utilisation d'une partie de la production obtenue comme semences pour installer les cycles suivants de culture.

${ }^{2}$ Le paiement de la redevance d'emploi est généralement justifié par les investissements spécifiques dans le domaine des biotechnologies. On ne peut cependant comprendre le niveau élevé de la redevance sans connaître les sommes d'argent engagées dans les opérations associées de partenariat entre les grandes firmes impliquées ni les motivations financières de ces dernières. A titre indicatif, Monsanto et Delta \& Pineland ont signé, le 8 Mai 1998, un accord permettant au premier d'absorber le deuxième. Cet accord a été invalidé par un jugement du 20 Décembre 1999 pour non-conformité à la loi anti-trust des Etats-Unis. Cet échec conduisit Delta \& Pineland à réclamer compensation des dommages et intérêts à Monsanto, d'un montant d'un milliard de US\$ (Edgaronline, 2005).
} 
Il y a une grande variation des niveaux de redevance exigée entre les pays où celle-ci s'applique. Cette variation est attribuée aux différences dans la réduction espérée d'utilisation des insecticides [12]. Plus élevée est l'espérance de réduction, plus coûteuse sera la redevance d'emploi de la technologie à payer. En d'autres termes, il est de bonne logique commerciale de vanter une forte économie en insecticides pour faire payer plus chère la technologie.

Le niveau de la redevance varie aussi dans le temps dans un même pays. Aux Etats-Unis, la redevance a augmenté alors qu'en Australie, les fermiers ont pu la faire baisser (cf. infra). Dans les pays où la redevance d'emploi ne s'applique pas, et mises à part les variétés hybrides, les différentiels de prix des semences sont d'un niveau plus faible que la redevance d'emploi. En Chine, le marché concurrentiel [13] semble bien induire une baisse du différentiel des prix de semences, mais cette tendance semble s'inverser de nouveau avec l'offre de variétés hybrides pour capter une rente plus confortable.

Tableau 2. Variation du niveau de la redevance d'emploi et du différentiel de prix des semences de coton Bt (US\$/ha)

\begin{tabular}{|c|c|c|c|c|c|c|c|c|c|}
\hline & \multicolumn{5}{|c|}{ Redevance d'emploi } & & \multicolumn{3}{|c|}{ Différentiel de prix des semences (Bt - non-Bt) } \\
\hline & \multirow[b]{2}{*}{ USA } & \multirow[b]{2}{*}{ Australia } & \multicolumn{2}{|c|}{ South Africa } & \multirow[b]{2}{*}{ Mexique } & \multirow[b]{2}{*}{ Argentine } & Inde & \multicolumn{2}{|c|}{ Chine } \\
\hline & & & Dry, small & Irrigate, Large & & & Hybrides & Population & Hybrides \\
\hline $\begin{array}{l}1996 / 97 \\
1997 / 98\end{array}$ & 66 & 135 & & & 1 & & & & \\
\hline $1998 / 99$ & & & & & 56 & & & & \\
\hline $1999 / 00$ & & & & & & 75 & & 38 & \\
\hline 2000/01 & & & & & & 85 & & 60 & \\
\hline 2002/03 & 80 & & 49 & 104 & & & 21 & & \\
\hline 2003/04 & & 85 & & & & & & & \\
\hline 2004/05 & & & & & & & & 5 & 83 \\
\hline
\end{tabular}

La diffusion du CGM a associé dans la pratique Monsanto, firme de biotechnologie, et Delta \& Pineland Company, firme semencière disposant d'un certain portefeuille variétal. Il a découlé de ce partenariat, s'agissant du coton, la conséquence d'une approche mondiale de la diffusion d'un nombre limité de variétés de CGM. La variété NUCOTN 33B par exemple a été commercialisée aux Etats-Unis, en Mexique, en Argentine, en Afrique du Sud et en Chine. Aux Etats-Unis et en Australie, les variétés respectivement appelées Bollgard et Ingard sont identiques. Cette démarche est discutable sur le plan agronomique car il est irréaliste d'attendre qu'un nombre limité de variétés puisse convenir à la diversité de conditions naturelles d'utilisation. La moindre performance, quantitative et/ou qualitative, de ces variétés a en effet été observée en Chine et au Mexique [14, 15].

On recense actuellement deux pays où la diffusion du CGM se fait sans que les paysans aient à payer une redevance d'emploi. L'exemple le plus significatif est la Chine où les paysans n'ont même pas à s'engager formellement en signant un contrat [13]. Le coût de la technologie est alors intégré dans le prix de semences. Les paysans chinois ont cependant conservé la liberté d'utiliser les graines de leur production pour servir de semences, ce dont ils ne se privent pas. Dans les provinces utilisant essentiellement les variétés conventionnelles de CGM ("population", non hybride), près de $50 \%$ des paysans ne renouvellent pas leurs semences tous les ans [13].

Le même cas de figure s'applique aux paysans en Inde depuis la commercialisation en 2002, mais avec la grande différence d'une diffusion exclusive de variétés hybrides F1 dont les semences doivent être renouvelées annuellement. Ce n'est pas forcément le cas [16], et cela pourrait expliquer les grandes différences des résultats observés [17].

\section{II.2. Innovation institutionnelle en évolution}

La brouille quasi consommée entre les deux alliés [18], Monsanto et Delta \& Pineland Company, semble accentuer une variation dans l'approche de Monsanto. Ce dernier est plus volontiers désireux de donner licence à l'utilisation de ses gènes dans les variétés 
développées par les pays d'utilisation. C'est le cas en Inde $^{3}$ mais dans une démarche commerciale de diffusion exclusive de variétés hybrides.

Le coût de la technologie est alors intégré directement dans le prix des semences. On constate aussi que le nombre de fournisseurs, essentiellement locaux, de variétés de CGM augmente [19]. La situation de monopole au profit d'une firme étrangère ne s'applique donc pas aux semences mais à la fourniture des gènes.

Les firmes biotechnologiques ajustent donc les innovations institutionnelles qui accompagnent l'utilisation du CGM, pour préserver leur rente biotechnologique. Cela est général dans la diffusion des OGM. Au Brésil, Monsanto a réussi à faire intervenir les négociants nationaux de soja comme collecteurs de royalties à son profit [20].

\section{III.Des résultats complexes mais porteurs d'enseignements}

Il n'est pas aisé de faire une synthèse des résultats rapportés sur l'utilisation du coton Bt dans le monde. Les résultats peuvent être obtenus en culture pluviale ou irriguée, dans des exploitations de tailles très variables, pour des années différentes, pour des régions différentes d'un même pays, pour des pays à niveaux de développement économique très distincts. Le cas de l'Afrique du Sud est typique, avec à la fois petite agriculture familiale (noire) et agriculture de grandes fermes (blanches), culture irriguée ou pas.

En dépit des réserves formulées ci-dessus, il est utile de réunir les résultats pour en faciliter la synthèse. Nous l'avons fait, de manière encore imparfaite et incomplète, en réunissant les résultats des pays suivant la taille des exploitations et l'irrigation, un pays comme l'Afrique du Sud pouvant se retrouver dans les deux groupes de pays (Tableau 3 et Tableau 4).

\section{III.1. Des résultats à considérer dans la limite des méthodes mises en oeuvre}

Les méthodes par lesquelles les résultats sont obtenus et rapportés sont aussi fréquemment discutables. Les résultats présentés par Qaim Matin [21], ayant contribué à l'idée d'une très forte rentabilité du coton Bt en Inde, correspondent surtout à une extrapolation de résultats d'expérimentation fournis par la firme Monsanto. Lorsque les résultats sont obtenus effectivement en situation d'utilisation réelle chez les paysans, les comparaisons de rentabilité ont rarement lieu entre des parcelles de coton Bt et de coton conventionnel cultivées dans les mêmes fermes. Même quand cela est le cas, les variétés de coton Bt et de coton conventionnel ne se différencient pas seulement par ce seul caractère (on dit qu'elles ne sont pas isogéniques). Il est donc abusif d'attribuer les différences observées au seul caractère $\mathrm{Bt}$ comme cela est couramment fait.

Il y a donc une difficulté technique pour une méthode irréprochable de comparaison, et cette difficulté est accentuée par la rapidité de diffusion du coton Bt dans certains pays (en Chine notamment) : on y trouve très peu ou pas de parcelles de référence en coton conventionnel.

D'autres biais sont par contre plus directement liés à l'approche méthodologique du chercheur. Le choix de la méthode de budget partiel conduit à supposer, souvent, que seuls les postes de dépense en semences et en protection phytosanitaire sont modifiés. Cette hypothèse s'avère erronée car on constate que les paysans tendent à intensifier davantage, et à accorder plus de soin, lorsque l'espérance de rendement est augmentée par l'utilisation du coton Bt. Cela est observé dans plusieurs pays et notamment en Inde [22].

La surestimation peut découler aussi de l'hypothèse d'un prix identique à la vente du coton $\mathrm{Bt}$ et du coton conventionnel. Cette hypothèse est discutable car la qualité de la fibre du coton $\mathrm{Bt}$ peut être moins bonne pour diverses raisons, comme au Mexique [15] ou en Inde [22]. Aux

\footnotetext{
${ }^{3}$ Mahyco Monsanto Biotech (India) Ltd est une entreprise à capitaux mixtes par laquelle Monsanto donne licence aux firmes semences en Inde. En 2005, licence a été donnée à 19 firmes semencières à travers le pays.
} 
Etats-Unis, la moindre qualité du CGM a été mise en cause obligeant Monsanto à se défendre [23].

Une autre erreur d'estimation interpelle davantage car elle est plus systématique dans les pays où les parcelles refuges sont imposées (très peu protégées contre les ravageurs). Le rendement obtenu sur ces parcelles refuges est obligatoirement bien plus faible. Or ces parcelles sont rarement prises en compte dans la comparaison de rentabilité, ce qui surestime l'impact économique du gène Bt.

La grande variation de résultats entre les pays et à l'intérieur d'un même pays tend à alimenter surtout les polémiques. Pourtant, l'application de toute nouveauté technique ne peut pas conduire qu'à des résultats uniformes. Pour cerner les perspectives d'extension de l'utilisation du coton $\mathrm{Bt}$, l'analyse de l'hétérogénéité des résultats, et des facteurs responsables, est fondamentale. Par commodité, la discussion des résultats est organisée en fonction des deux groupes, avec les limites que cela comporte.

\section{III.2. Incidence négligeable du gain de productivité en grande production irriguée}

Dans le groupe de pays aux exploitations agricoles de grande taille, le recours à l'irrigation explique la grande variation dans les rendements obtenus (Tableau 3). Cela est manifeste en Afrique du Sud mais aussi aux Etats-Unis où la production peut être irriguée ou pas du tout. En Argentine, la production n'est pratiquement jamais irriguée, le niveau de rendement est bien plus faible qu'en Australie où l'irrigation est systématique.

Tableau 3. Rentabilité comparée du coton Bt en culture cotonnière à grande échelle des pays industrialisés ou émergents

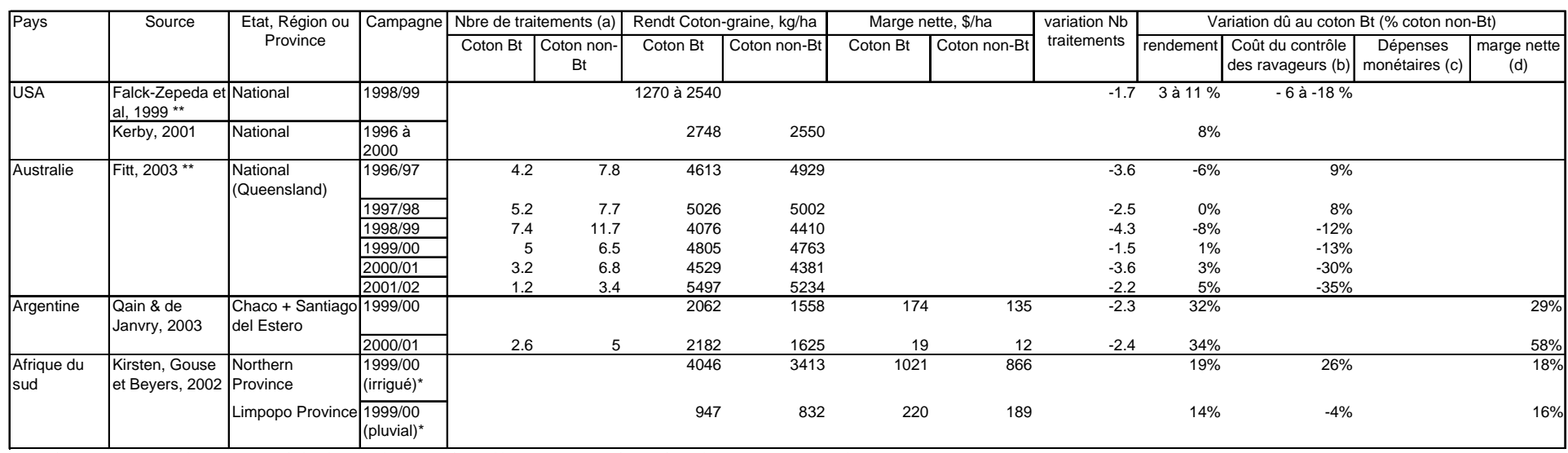

列

(b) coût des semences + coût des insecticides ; (c) Dépenses pour les intrants et la main-d'œuvre salariée ; (d) Marge brute - dépenses monétaires

* Marge nette avec seulement déduction coût semences et insecticides

** variation du coût des intrants en tenant compte seulement du poste de contrôle des ravageurs (semences + insecticides)

Aux Etats-Unis, en Australie mais aussi partiellement en Afrique du Sud, la production intensive dans l'usage des intrants conduit à des rendements élevés. C'est une production très capitalistique. Le temps consacré à la culture d'un hectare aux Etats-Unis peut être estimé à douze heures/ha (à comparer aux 100-150 jours/ha dans les pays de l'Afrique Zone Franc). Cette indication permet de souligner que le gain de productivité (du travail) dans ces pays découle plus de l'augmentation du rendement et/ou de la réduction des coûts de production.

Dans ces pays, le gain de rendement est faible voire nul et le nombre de traitements insecticides est réduit seulement de 2 à 3 (Tableau 3). En Australie, il n'y a pratiquement pas de gain de rendement. La réduction du coût dans le contrôle des ravageurs est à peine perceptible [24]. Aux Etats-Unis, les résultats (issus essentiellement des travaux d'expérimentation) sont très variés, car les conditions de pression parasitaire diffèrent entre les Etats. En moyenne, le gain de rendement serait de 3-11\%, avec un niveau de rendement peu élevé. Les réductions de coûts de contrôle des ravageurs sont aussi de cet ordre (6-18\%). Globalement, le gain de productivité n'est pas élevé. 
On peut même se demander si ce gain existe bien au regard des statistiques générales fournies par l'USDA ${ }^{4}$, et basées sur les éléments en conditions réelles de production. Par rapport à la période avant l'adoption du CGM, sur une période de dix ans après la commercialisation du CGM, le rendement moyen n'a été supérieur que lors des deux dernières années (Figure 1). Les coûts de production à l'hectare ont augmenté car les coûts consacrés aux semences ont fortement augmenté, sans que ceux des pesticides (insecticides, herbicides...) aient diminué (Figure 2). Le coût unitaire de production n'a réellement baissé que pour les deux dernières campagnes sur une période de dix ans (Figure 3).

Figure 1. Evolution du rendement aux Etats-Unis, 1975-2004

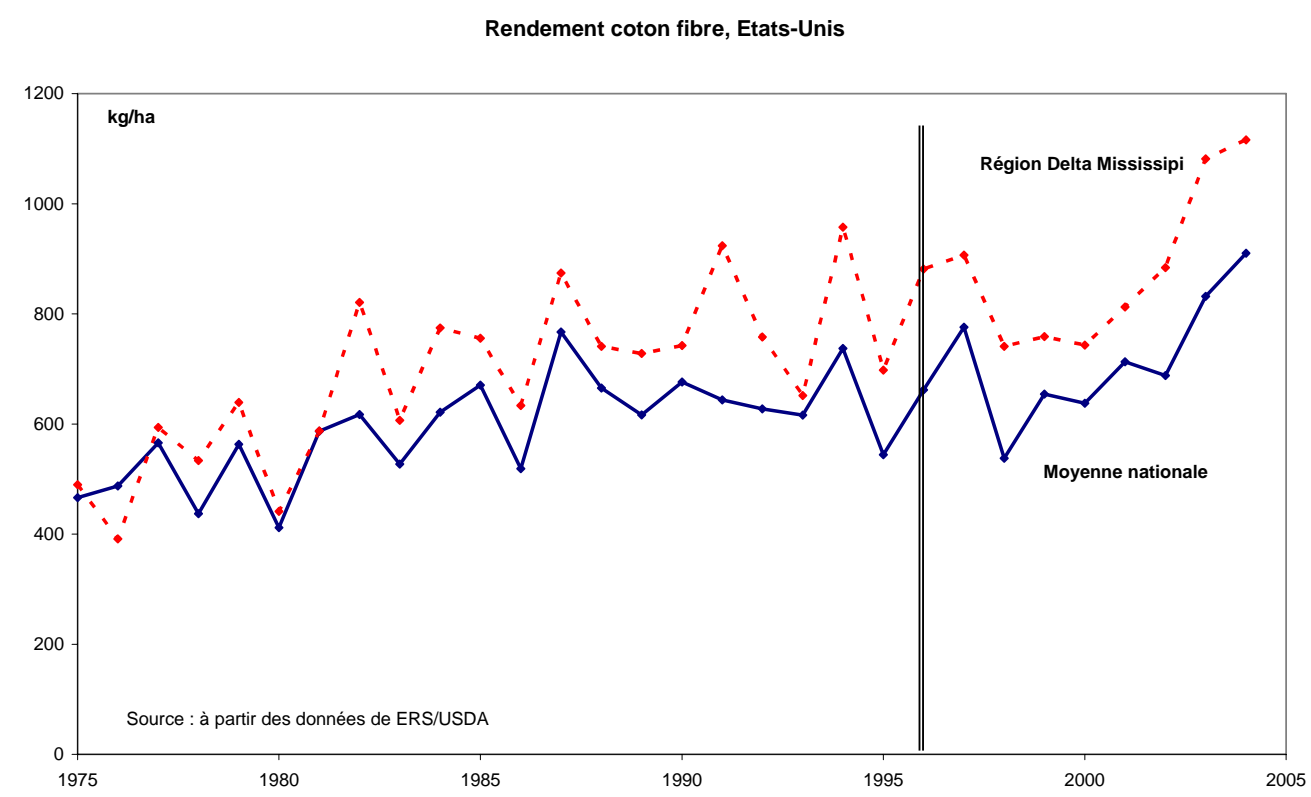

Note : A côté de la moyenne nationale, on a fait apparaître celle de la région du Delta de Mississipi où l'utilisation du cotonBt est plus forte.

Des enquêtes réalisées aux Etats-Unis, en Australie et auprès des fermiers blancs de l'Afrique du Sud, il ressort clairement la notion de "confort" procuré par le coton Bt dans le contrôle des ravageurs. Les fermiers sont moins soumis à la contrainte du comptage des chenilles pour les traiter chimiquement aux stades où elles sont les plus sensibles. Le soulagement de cette contrainte est appréciable quand elle intervient à la période de Noël et des vacances d'été, comme c'est le cas en hémisphère sud [25]. En Australie, au "confort" mentionné s'ajoute la bonne conscience. Face à la pression de la société civile contre l'usage jugé excessif des pesticides, la réduction du nombre de traitements résultant du coton Bt permet aux planteurs de se sentir moins mal à l'aise face aux critiques écologistes [26].

Figure 2. Evolution des coûts des semences et des pesticides aux Etats-Unis, 1975-2004

\footnotetext{
${ }^{4}$ Le Economic Research Service de l'USDA fournit régulièrement des Farm Business Economics Report sur les principales productions agricoles du pays avec les statistiques sur les coûts de production.
} 
Coût des semences et des pesticides, \$/acre. Région Delta/Mississipi Portal, Etats-Unis

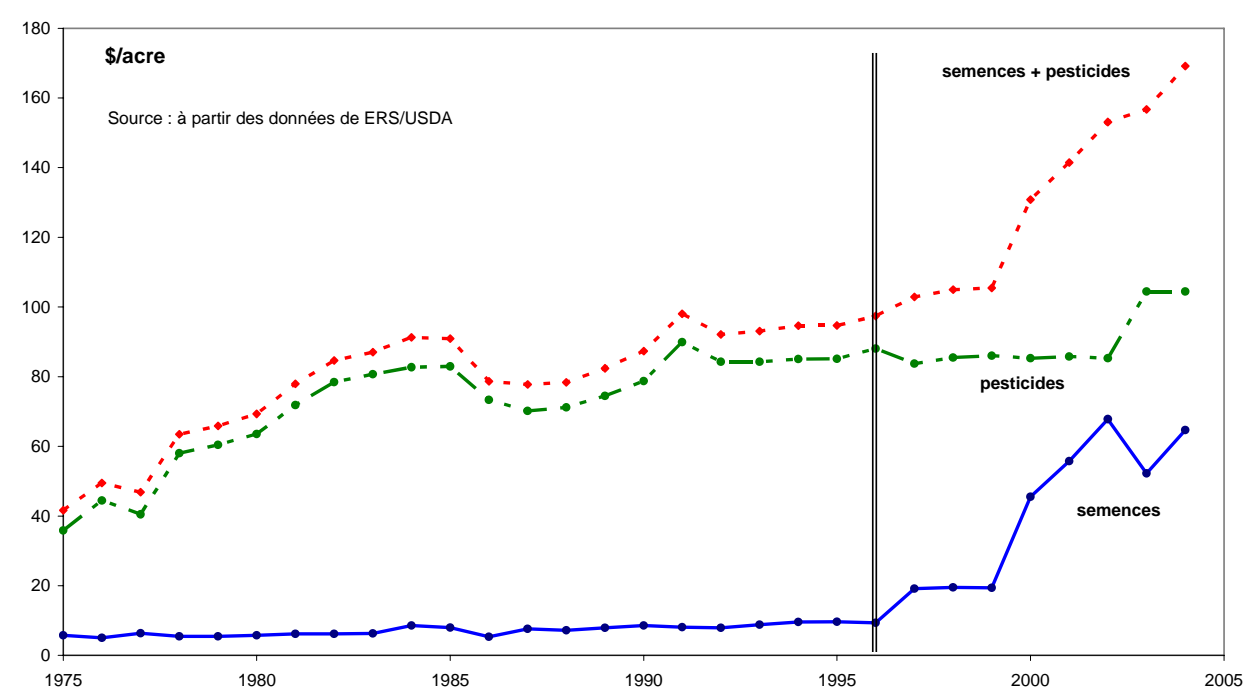

En production pluviale, les niveaux de rendement peuvent varier beaucoup entre les pays, en fonction des pluviométries mais aussi en fonction du degré d'intensification des cultures. Avec un niveau de rendement plus élevé, résultat d'une intensification plus poussée, la rentabilité à l'hectare est plus faible en Argentine qu'en Afrique du Sud. Cette rentabilité est aussi très dépendante d'une baisse du prix d'achat du coton produit (cas de l'année 2001). Dans ces conditions, l'intérêt économique du coton Bt est douteux si l'on se réfère aux valeurs de marges nettes (moins de 20 \$/ha). L'indication d'un gain relatif important en marge nette du coton Bt (ici 58\%) peut être trompeuse, car le gain en valeur absolue peut être très faible.

Figure 3. Evolution du coût unitaire de production de coton fibre aux Etats-Unis, 1975-2004

Coût total, \$llivre. Etats-Unis

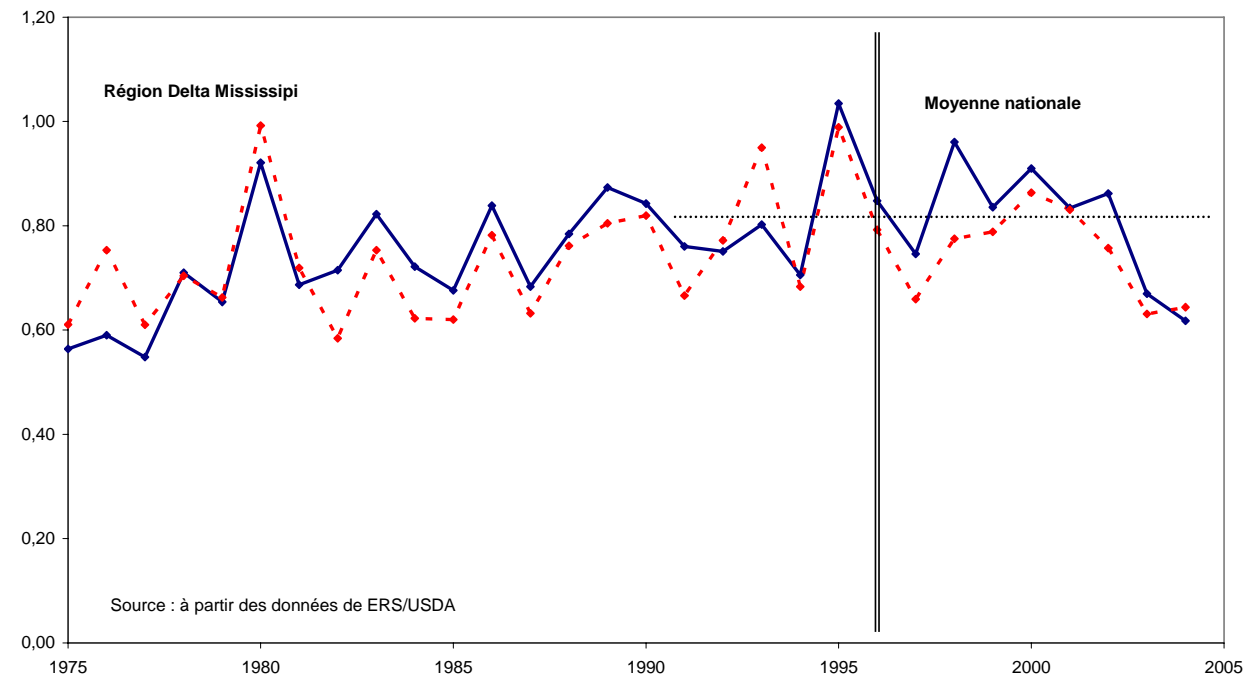

\section{III.3. Effets variables aux facteurs multiples dans les petites exploitations}

Dans les pays où le coton Bt est utilisé dans des exploitations familiales de petites tailles, les effets sont globalement positifs mais avec une grande variation du sens et de l'amplitude des effets observés. Une telle variation peut donner lieu à une grande divergence d'appréciation. Le cas le plus controversé concerne l'utilisation de semences hybrides de coton-Bt dans l'Etat 
d'Andhra Pradesh en Inde, réussite exemplaire pour certains -ISAAA ${ }^{5}$, ICAC $^{6}$, Monsanto, firmes semencières internationales ou indiennes- mais aussi des économistes spécialisés dans le suivi des OGM [27] - échec cuisant pour d'autres (ONG indiennes et internationales relayées par les media) [28]. Il doit néanmoins y avoir problème : le Gouverneur de cet Etat vient de sommer la firme Mahyco ${ }^{7}$ (qui a fourni les semences) de dédommager les paysans [29]. Les causes possibles du problème sont cependant diverses (inefficacité des gènes utilisés, manque de formation et d'information des paysans, mauvaise qualité des semences de contrefaçon...). Cet exemple témoigne que l'avantage économique de l'utilisation du coton $\mathrm{Bt}$ dans un PED ou pays émergent est sous l'influence de facteurs autres que le caractère transgénique. On peut citer par exemple le facteur organisationnel dont on verra le rôle crucial dans la distribution des semences.

\section{Portée relative de l'efficacité et des externalités}

Partout où le coton Bt est utilisé, on observe une réduction du coût des insecticides utilisés pour contrôler les ravageurs du cotonnier (Tableau 4). Cette réduction est variable entre les pays, entre les années dans un même pays, comme en Afrique du Sud [30] ou entre les régions d'un même pays comme en Chine. Le degré de réduction dépend du niveau de pression parasitaire avant le recours au coton $\mathrm{Bt}$ et du nombre de traitements chimiques que les paysans appliquaient alors. Il en découle que la réduction du nombre de traitement, et donc celle du coût correspondant, est la plus forte en Chine, du moins dans les provinces situées le long de la vallée du Fleuve Jaune [31], mais pas partout dans ce pays [14].

Tableau 4. Rentabilité comparée du coton Bt en culture cotonnière à petite échelle des PED ou pays émergents

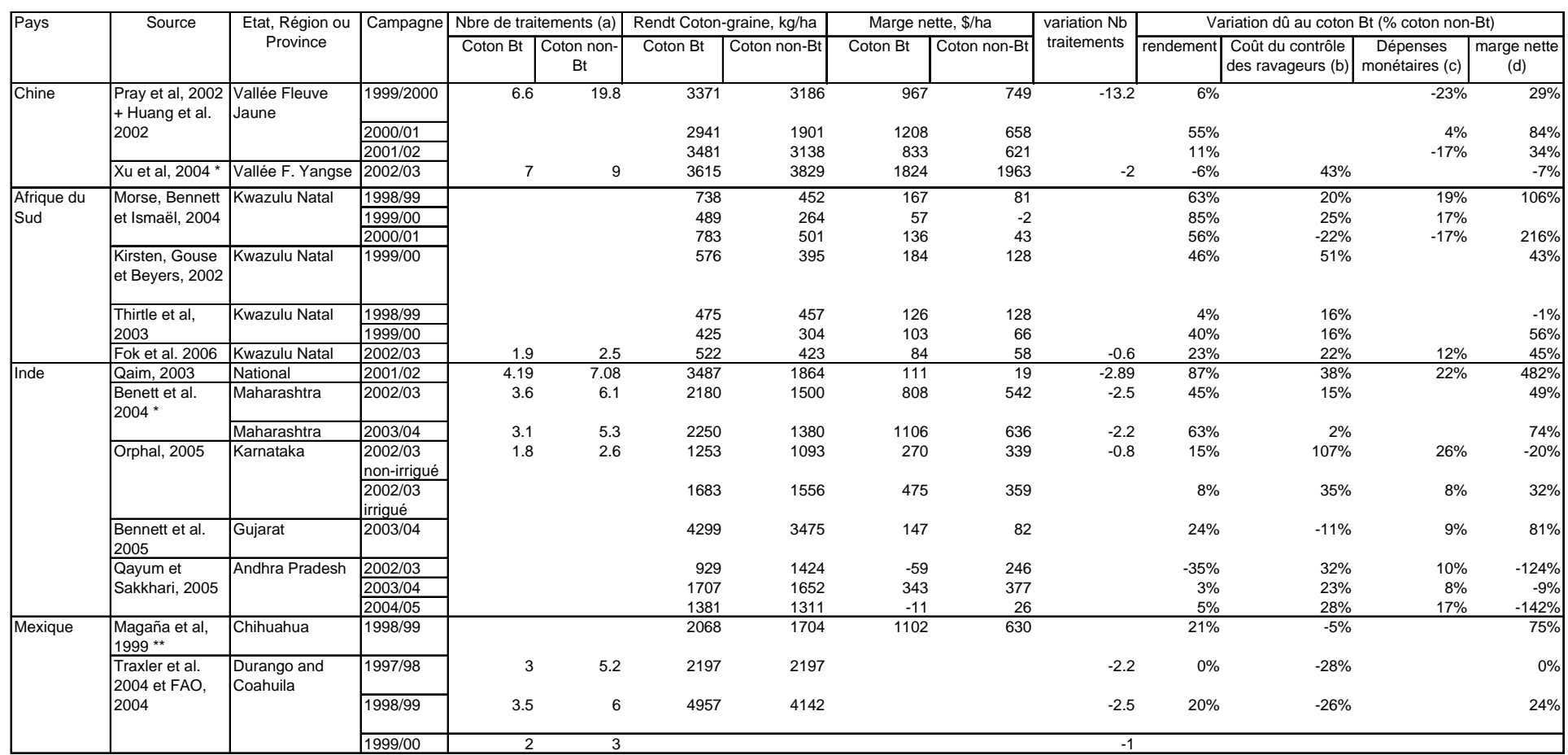

(a) Traitements contre tous les ravageursmais les données de Pray et al. en Chine concernent seulement les traitements contre H. armigera

(b) coût des semences + coût des insecticides ; (c) Dépenses pour les intrants et la main-d'œuvre salariée ; (d) Marge brute - dépenses monétaires

* Marge nette avec seulement déduction coût semences et insecticides

** variation du coût des intrants en tenant compte seulement du poste de contrôle des ravageurs (semences + insecticides)

note : Mexique, résultats de Traxler, différence de marge nette prenant en compte seulement les coûts de semences et de pesticides

\footnotetext{
${ }^{5}$ International Service for the Acquisition of Agri-biotech Applications

${ }^{6}$ International Cotton Advisory Committee

${ }^{7}$ Mahyco Monsanto Hybrid Company est une joint venture associée à Monsanto.
} 
Dans aucun pays, les traitements insecticides n'ont disparu. Les toxines produites par le coton Bt ne sont efficaces que contre certains types de ravageurs cibles (les chenilles des lépidoptères, surtout Helicoverpa armigera mais aussi Pectinophora gossypiella, Earias sp.), pendant une partie du cycle du cotonnier et si les conditions climatiques ne nuisent pas à l'expression du gène $\mathrm{Bt}$. Il reste donc nécessaire de traiter, un peu, contre les ravageurs cibles. Il est aussi normal de constater que le nombre de traitements chimiques contre les ravageurs non-cibles du coton Bt ne diminue pas. Ce nombre peut même augmenter, comme en Afrique du Sud et surtout en Chine [13, 32].

La réduction du coût total de protection phytosanitaire, résultant du coût des insecticides et des semences de coton $\mathrm{Bt}$, est loin d'être systématique. Ce coût total est même fréquemment plus élevé avec le coton Bt, car le surcoût des semences n'est pas compensé par l'économie découlant de la diminution du nombre de traitements chimiques.

La réduction du nombre de traitements insecticides est généralement associée à un effet bénéfique pour la santé des paysans. Cette perception est à nuancer [30, 33]. Les produits insecticides que le coton Bt permet d'économiser (les pyréthrinoïdes) ont une toxicité faible par rapport aux produits organophosphorés qu'il faut utiliser, parfois en plus grande quantité, pour traiter contre les ravageurs non-cibles.

La réduction du nombre de traitements insecticides par le coton $\mathrm{Bt}$ se traduit par une diminution du travail et de la pénibilité du travail associé. L'ampleur de cette réduction est cependant souvent exagérée. Pour la Chine, Huang et al. signalent une économie en travail de plus de 80 jours/ha [31] mais c'est en retenant une hypothèse irréaliste d'un travail de cinq jours pour traiter un hectare de coton.

S'agissant du coton Bt, il est important de rappeler que l'effet positif sur le rendement n'était pas avancé lors de son lancement en 1996. L'intérêt anticipé par les promoteurs de ce coton était la diminution du coût de production par la réduction du nombre de traitements insecticides. L'augmentation du rendement observé dans certains cas était donc une bonne surprise. Cette augmentation est cependant variable entre les pays et entre les années. Pour l'Afrique du Sud, les gains très importants observés dans les premiers travaux [34] n'ont pas été confirmés dans des travaux plus récents [11,30]. C'est dans les petites exploitations des PED où le gain de rendement observé, en valeur relative, est le plus élevé. Il y a en fait une relation inverse entre le niveau initial et le gain (relatif) de rendement. C'est quand le niveau initial est faible que le taux de gain est plus élevé. Ce point est intéressant et il est favorable aux paysans dont le niveau de rendement est pénalisé par diverses contraintes structurelles de leurs exploitations.

Le rendement du cotonnier dépend du contrôle des ravageurs. Globalement, l'absence de traitements chimiques fait perdre $35-41 \%$ du rendement potentiel mais il y a une perte de rendement de 7-24\% même quand le contrôle chimique des ravageurs est appliqué [35]. Dans la réalité, il est techniquement impossible de traiter systématiquement au meilleur escient au moment où les chenilles sont aux stades les plus sensibles, et de manière à les atteindre partout où elles peuvent se nicher. A titre indicatif, les produits chimiques peuvent être lavés par les pluies qui surviennent après leur épandage.

Les traitements chimiques ne sont donc pas parfaitement efficaces et ne peuvent pas l'être. Il y a forcément un manque à gagner par rapport au rendement potentiel et qui est d'autant plus grand que le contrôle chimique est insuffisant ou mal réalisé. L'utilisation du coton Bt permet en fait de récupérer partiellement le manque à gagner et de s'approcher davantage du rendement potentiel. Dans beaucoup de PED, il y a de nombreuses raisons qui empêchent la réalisation correcte de la protection chimique et que les paysans ne peuvent pas contrôler (absence de crédit, défaillances du réseau de distribution des intrants...). L'utilisation du coton Bt réduit ainsi la dépendance des paysans aux imperfections de ce type de contexte et cela 
constitue indéniablement un élément positif.

\section{Rentabilité variable et sous dépendance}

Le gain de rentabilité procuré par l'utilisation du coton Bt est variable et il dépend fondamentalement de quatre facteurs : le surcoût des semences, le degré de réduction du coût des insecticides, le degré de gain de rendement, le niveau et la stabilité du prix de vente du coton-graine. Le premier facteur est le seul qui dépend des conditions de distribution des semences de coton Bt mais il focalise la méfiance des opposants aux OGM. Les autres facteurs sont dépendants des conditions des pays utilisateurs et méritent aussi d'être pris en compte dans les réflexions sur les conditions de rentabilité du coton Bt.

Lorsque le surcoût des semences n'est pas trop élevé et qu'il est facilement compensé par la réduction du coût des insecticides, qu'un gain de rendement est enregistré, avec un produit qui ne souffre pas d'une décote de prix, on a la situation idéale d'un gain de rentabilité indéniable. Une telle combinaison favorable des quatre facteurs est cependant assez rare. La Chine bénéficie quasiment de cette combinaison favorable mais dans beaucoup d'autres PED, le gain de rentabilité n'est pas si élevé parce que le coût du contrôle des ravageurs n'a pas diminué, quand il n'a pas augmenté.

Ces observations justifient la méfiance des opposants aux OGM car la cherté des semences pénalise les paysans utilisateurs. Cette cherté pénalise aussi les fournisseurs de semences car l'extension de l'utilisation du CGM s'en trouve limitée [36] et que les utilisateurs sont encouragés à tricher pour ne pas payer la redevance d'emploi jugée trop élevée [37].

Dans une année donnée, au niveau d'un paysan, l'espérance de la rentabilité du coton Bt dépend de celle du rendement. Or l'espérance de rendement en production pluviale, et en petit paysannat, peut être limitée pour des raisons incontrôlables du domaine climatique (installation tardive des pluies par exemple), du domaine institutionnel (approvisionnement défaillant en semences ou en intrants dans une filière déstabilisée), ou pour des raisons propres à l'exploitation (manque de main-d'oeuvre). Cela est vrai même sans recours aux CGM mais avec l'utilisation de semences CGM nécessitant un débours monétaire important en début de saison, l'incidence négative sur la rentabilité est plus forte.

Cette analyse met en évidence le danger économique à prôner l'utilisation exclusive du CGM comme cela est implicite dans le débat actuel.

\section{IV.Les éléments à gérer pour une extension bénéfique du coton Bt dans les PED}

Le débat sur la pertinence des OGM pour les PED oppose des effets anticipés par les partisans et opposants que l'on peut résumer dans le cas du coton Bt (Tableau 5).

Tableau 5. Synthèse sur les effets anticipés relatifs à l'utilisation du coton Bt

\begin{tabular}{|l|l|}
\hline Effets positifs & Effets négatifs \\
\hline Coton Bt efficace contre les ravageurs cibles & Non efficacité contre les ravageurs cibles \\
\hline Réduction de l'usage des insecticides & Coût élevé des semences pour les utilisateurs \\
\hline Réduction du coût de contrôle des ravageurs & $\begin{array}{l}\text { Effet de barrière à l'utilisation de semences pour } \\
\text { beaucoup de paysans }\end{array}$ \\
\hline Rentabilité améliorée & $\begin{array}{l}\text { Instauration d'une situation de monopole des } \\
\text { semences de coton Bt }\end{array}$ \\
\hline Contribution à la réduction de la pauvreté & $\begin{array}{l}\text { Obligation du renouvellement annuel des semences } \\
\text { qui accentue les coûts de production }\end{array}$ \\
\hline Réduction du travail et de sa pénibilité & Effets néfastes sur la faune non-cible \\
\hline Effet positif sur la santé & $\begin{array}{l}\text { Induction d'une résistance des ravageurs à la toxine } \\
\text { Bt }\end{array}$ \\
\hline & $\begin{array}{l}\text { Impossibilité pour le coton non GM de co-exister } \\
\text { avec le coton Bt }\end{array}$ \\
\hline & $\begin{array}{l}\text { Diffusion incontrôlée du gène Bt avec des effets } \\
\text { indésirables }\end{array}$ \\
\hline
\end{tabular}


A la lumière des résultats rapportés ci-dessus, on peut donner une vision plus complète des effets de l'utilisation du coton-Bt. Les effets anticipés ne sont pas tous confirmés ou de manière systématique (Tableau 6).

Tableau 6. Des effets imparfaitement anticipés de l'utilisation du coton-Bt

\begin{tabular}{|c|c|c|}
\hline Effets positifs & Effets neutres & Effets négatifs \\
\hline $\begin{array}{l}\text { Efficacité avérée du coton Bt } \\
\text { contre les ravageurs cibles }\end{array}$ & $\begin{array}{l}\text { Nuisance non établie sur la faune } \\
\text { non-cible }\end{array}$ & $\begin{array}{l}\text { Instauration fréquente d'une } \\
\text { situation de monopole pour les } \\
\text { semences de CGM }\end{array}$ \\
\hline $\begin{array}{l}\text { Réduction avérée de l'usage des } \\
\text { insecticides contre les ravageurs } \\
\text { cibles, mais réduction seulement }\end{array}$ & $\begin{array}{l}\text { Nuisance non-établie sur les } \\
\text { prédateurs des ravageurs }\end{array}$ & $\begin{array}{l}\text { Chamboulement des services } \\
\text { semenciers établis }\end{array}$ \\
\hline $\begin{array}{l}\text { Réduction du coût des } \\
\text { insecticides, mais réduction } \\
\text { seulement }\end{array}$ & $\begin{array}{l}\text { Emergence non avérée de } \\
\text { résistance des ravageurs cibles } \\
\text { aux toxine } \mathrm{Bt}\end{array}$ & $\begin{array}{l}\text { Obligation quasi systématique du } \\
\text { renouvellement annuel des } \\
\text { semences }\end{array}$ \\
\hline $\begin{array}{l}\text { Rentabilité fréquemment } \\
\text { améliorée mais à des degrés très } \\
\text { variables }\end{array}$ & $\begin{array}{l}\text { Peu d'éléments pour statuer sur la } \\
\text { Co-existence (CGM et coton } \\
\text { conventionnel) }\end{array}$ & $\begin{array}{l}\text { Coût élevé des semences de } \\
\text { manière très fréquente }\end{array}$ \\
\hline & & $\begin{array}{l}\text { Réduction du coût total du contrôle } \\
\text { des ravageurs est exceptionnelle }\end{array}$ \\
\hline
\end{tabular}

Il y a aussi un grand nombre d'effets non réellement anticipés, qui peuvent d'ailleurs être positifs ou négatifs (Tableau 7). Il ressort que, en dépit de l'attention que le sujet avait attirée, l'anticipation des effets a été très incomplète, en dépit des connaissances disponibles.

On observe un excès de pessimisme ou d'optimisme dans les deux catégories d'effets, comme cela ressort de l'analyse qui suit. 
Tableau 7. Des effets incomplètement anticipés de l'utilisation du coton-Bt

\begin{tabular}{|l|l|}
\hline Effets positifs & Effets négatifs \\
\hline Gain de rendement, mais à un degré variable & $\begin{array}{l}\text { Changement de la structure des coûts de production } \\
\text { accentuant le risque financier de l'utilisation des } \\
\text { semences de CGM }\end{array}$ \\
\hline $\begin{array}{l}\text { 'confort' dans la réalisation du contrôle contre les } \\
\text { ravageurs }\end{array}$ & $\begin{array}{l}\text { Effets pervers des comportements opportunistes pour } \\
\text { ne pas payer la redevance d'emploi des semences }\end{array}$ \\
\hline $\begin{array}{l}\text { Affranchissement des incertitudes d'obtention des } \\
\text { insecticides pour les paysans dans de nombreux PED }\end{array}$ & $\begin{array}{l}\text { Accentuation du coût des semences pour des } \\
\text { changements de conditions de distribution des } \\
\text { semences pour mieux préserver la rente } \\
\text { biotechnologique }\end{array}$ \\
\hline $\begin{array}{l}\text { Retour possible à un niveau bien plus facilement } \\
\text { gérable de la pression parasitaire }\end{array}$ & $\begin{array}{l}\text { Grande variation de la rentabilité entre les pays, entre } \\
\text { les régions d'un pays, entre les années, et entre les } \\
\text { producteurs }\end{array}$ \\
\hline $\begin{array}{l}\text { Capacité, quoique rare, des utilisateurs à intégrer le } \\
\text { coton Bt dans une approche intégrée de contrôle des } \\
\text { ravageurs }\end{array}$ & $\begin{array}{l}\text { Danger économique de la couverture à 100\% en } \\
\text { CGM }\end{array}$ \\
\hline $\begin{array}{l}\text { Il y a une marge pour la négociation des conditions } \\
\text { de distribution et d'utilisation des semences de CGM }\end{array}$ & Danger écologique de la couverture à 100\% en CGM \\
\hline $\begin{array}{l}\text { Il y a une marge pour la négociation du coût } \\
\text { d'utilisation des semences de CGM }\end{array}$ & $\begin{array}{l}\text { Changement défavorable de la composition du } \\
\text { complexe parasitaire }\end{array}$ \\
\hline
\end{tabular}

\section{IV.1. Optimisme et pessimisme excessifs non avérés}

Les effets anticipés, positifs ou négatifs, ne sont pas confirmés de manière systématique (Tableau 6). On constate aussi que l'anticipation a été très incomplète car de nombreux effets n'avaient pas été pressentis (Tableau 7). Il ressort qu'il y a un continuum d'effets, mal anticipés, allant des bonnes surprises (des effets positifs non prévus) aux menaces non soupçonnées (effets négatifs non prévus), en passant par des effets anticipés plus ou moins confirmés.

S'agissant des effets anticipés initialement, force est de constater que, il n'y pas de confirmation complète et systématique, que les effets soient positifs ou négatifs. Les effets anticipés non avérés forment ainsi un troisième groupe d'effets neutres.

S'agissant des effets positifs anticipés, l'efficacité du coton Bt contre les ravageurs qu'il est censé contrôler est peu contestable. Elle se traduit par la réduction du nombre de traitements insecticides, de l'ordre de 2-3. Les traitements insecticides restent nécessaires, pour contrôler les ravageurs cibles du coton Bt et les ravageurs non-cibles. Il n'y a qu'une réduction du nombre de traitements chimiques sans forcément induire une diminution du coût total du contrôle (quand on combine le coût des semences et celui des traitements insecticides). Il en découle que l'amélioration de la rentabilité du coton Bt procède beaucoup du gain de rendement, plus fréquemment observé dans les PED, mais elle est souvent assez faible et fragile.

Cela est essentiellement dû à l'effet négatif d'une augmentation forte et générale du coût des semences. Comme cela a été craint, l'instauration d'un monopole dans la distribution des semences de coton Bt est quasi systématique, la Chine est une exception. Une telle situation de monopole a aussi provoqué l'effondrement du système semencier préexistant [37].

Par contre, il n'y a pas d'éléments pour justifier les craintes relatives aux impacts sur la faune non-cible. Il n'y a pas non plus de cas avérés d'apparition de résistance des ravageurs cibles contre la toxine Bt. On ne peut pas encore se prononcer sur l'impossibilité de faire du coton conventionnel dans les zones ayant adopté le coton Bt.

\section{IV.2. Avantages d'une certaine flexibilité méconnue}

La colonne de gauche du Tableau 7 indique que plusieurs effets positifs ne furent pas explicitement anticipés. Il n'est pas nécessaire de s'appesantir sur le gain de rendement, plus 
important, en termes relatifs, pour les paysans souffrant d'un niveau de rendement plus faible.

Tous les autres effets positifs non réellement anticipés révèlent un avantage de flexibilité procurée par le coton $\mathrm{Bt}$, notamment dans la pratique même du contrôle chimique des ravageurs, pour les grandes comme pour les petites exploitations.

Plus intéressant à souligner est la réversibilité des équilibres biologiques. L'utilisation du coton $\mathrm{Bt}$ en quelques années permet de revenir à un niveau de pression parasitaire plus faible et beaucoup plus facile à gérer, surtout en Chine où cette pression était parvenue à un niveau très élevé [13]. Bien sûr, un tel retour à une situation favorable peut ne pas durer si on n'y prend garde (cf. infra), mais l'observation de cette réversibilité est riche d'enseignements. L'hypothèse implicite sur l'occurrence brutale et irréversible des impacts environnementaux négatifs paraît ainsi discutable. Cela peut conduire à revoir les démarches actuelles de précaution, dont les coûts afférents (en temps et en termes financiers), notamment dans la conduite d'expérimentation de biosécurité, peuvent être jugés excessifs et insensés, et surtout pénalisants pour les PED [38].

Il faudrait peut-être oser manifester un peu plus de confiance dans la capacité de réaction des paysans qui, face à des signes d'évolution indésirable des équilibres biologiques, sont capables de changer de pratiques. Le cas des fermiers australiens réussissant à intégrer l'utilisation du coton $\mathrm{Bt}$ dans une démarche de lutte intégrée [24] est un témoignage positif dans ce sens. Certes, il serait hasardeux d'extrapoler l'acquis des 1200 fermiers australiens au grand nombre de paysans cotonniers des PED. C'est cependant une leçon intéressante pour aider les PED à se prémunir contre les messages qui tendent soit à encenser soit à diaboliser l'utilisation du coton Bt.

Enfin, un autre point positif se rapporte à une certaine variation des conditions d'utilisation du coton Bt. Tous les pays n'ont pas eu à se plier aux conditions d'utilisation imposées par Monsanto. Ni en terme d'engagement par contrat explicite et individuel, ni en terme de paiement de redevance d'emploi. Ni encore en terme d'acceptation sans négociation du niveau de cette redevance. La bonne nouvelle est donc qu'il y a un espace de négociation possible sur les conditions d'utilisation du coton Bt.

C'est fondamentalement ce niveau de redevance qui détermine pour beaucoup la rentabilité de l'utilisation du coton Bt. Il doit donc être négocié en fonction des espérances de gain que cette utilisation peut procurer. Plus on admet que les espérances de gain sont élevées, plus il faudra s'attendre à payer une redevance élevée. La surestimation de l'espérance est donc de bonne pratique commerciale, pour le commerçant. Pour le client, il doit prendre garde à une surestimation délibérée et infondée de l'espérance de gain.

\section{IV.3. D'important risques immédiats et du futur proche}

La colonne de droite du Tableau 7 met en évidence plusieurs niveaux de risque qui n'ont pas été explicités.

Le surcoût important des semences est porteur de risque financier élevé. Il se traduit par un changement de la structure des coûts de production [11,13] avec une forte augmentation de la part des dépenses à consentir dès l'installation de la culture. La perception du risque financier est ainsi accrue pour les paysans à ressources financières limitées. Dans cette situation, les paysans ont deux options de réaction : ne pas adopter ou adopter en trouvant des moyens pour réduire le coût du coton $\mathrm{Bt}$. Dans ce deuxième cas, une manière de faire est de réduire les doses d'utilisation. Une façon moins légale est de chercher à payer moins cher les semences, en puisant sur sa propre production même si on n'en a pas le droit, ou en achetant des semences illégales...

Ces comportements opportunistes peuvent induire des conséquences néfastes, certes pour les individus concernés, mais pas seulement. La détermination du surcoût des semences doit donc intégrer la capacité de contournement des paysans dans les PED et éviter que la "redevance ne 
tue la redevance" si elle est fixée à un niveau jugé inacceptable. Fixer ce niveau en fonction de l'espérance de gain des producteurs les moins performants serait une grosse erreur. 


\section{Dangers d'une couverture à $100 \%$ en coton Bt}

Une grande dispersion du rendement est un fait établi dans la production familiale des PED car beaucoup de facteurs empêchent les paysans à installer et à entretenir ses cultures de manière optimale. Chaque année, l'utilisation du coton Bt est forcément non rentable pour une partie des parcelles ou des paysans souffrant de rendements insuffisants.

En d'autres termes, il y a forcément une partie des parcelles qu'il ne faudrait pas faire ensemencer en coton Bt. Ce ne seront pas forcément les mêmes parcelles d'une année à l'autre, ni les mêmes paysans. Même si un PED a décidé d'adopter le coton Bt, la cohabitation avec du coton conventionnel est économiquement justifiée. La flexibilité doit être préservée pour que chaque paysan puisse décider, pour chacune de ses parcelles, d'utiliser ou pas le coton Bt en fonction des conditions climatiques et de ses contraintes personnelles. Pour que les paysans puissent appliquer cette flexibilité au mieux de leurs intérêts, ils doivent être informés sur les facteurs déterminant la rentabilité du coton Bt. A défaut, les résultats divergents obtenus par les paysans les amèneront à s'opposer dans la perception de l'intérêt du coton $\mathrm{Bt}$, à remettre en cause la décision relative à l'adoption de ce dernier et à douter de l'intégrité morale des décideurs.

Le raisonnement développé ici en faveur d'une flexibilité à accorder aux paysans s'oppose ainsi à l'hypothèse implicite d'une couverture à $100 \%$ en coton $\mathrm{Bt}^{8}$, tenue tant par les fournisseurs de coton Bt que par les opposants aux OGM. Sur le plan économique, l'abandon de cette hypothèse implicite aidera au débat sur les OGM, tout en posant un problème délicat de coordination dans une utilisation partielle.

La couverture à $100 \%$ en coton Bt n'est pas non plus justifiable sur le plan écologique. Le retour à un plus faible niveau de pression parasitaire, quelques années après l'utilisation du coton Bt, est un acquis positif mais fragile. Le souci de la préservation de ce faible niveau retrouvé s'oppose à une couverture à $100 \%$ en coton Bt qui accentue le risque de voir émerger effectivement la résistance des ravageurs aux toxines Bt.

La focalisation sur ce dernier risque paraît excessive. Elle est aussi trompeuse dans la mesure où elle a détourné l'attention sur les risques plus importants liés aux changements du complexe parasitaire. Dans ce domaine, ce n'est pas seulement une question de changement d'équilibre entre les familles de ravageurs, avec une importance accrue des insectes piqueurssuceurs par exemple [30]. Le changement intervenant entre les ravageurs d'une même famille est déjà observé, en Amérique latine et en Chine, et il paraît fort inquiétant. La chenille, Spodoptera litura (en Asie) ou litoralis (en Afrique et en Amérique), fort vorace, ne se contente plus de manger seulement les feuilles de cotonnier. Elle s'attaque aux organes fructifères [39] sans doute parce qu'il y a moins de chenilles de Helicoverpa armigera, très agressives, pour les en empêcher !

\section{Exigence en ajustement important de l'organisation de la distribution des semences}

L'adoption du coton Bt suivant une démarche de couverture partielle impose la nécessité d'une organisation adaptée de la distribution des semences. Dans les PED, comme c'est le cas de tous les pays au Sud du Sahara, la distribution des semences dans les villages est réalisée plusieurs mois avant le semis et avec une seule variété par village. Le principe de couverture partielle en coton $\mathrm{Bt}$ amène à réviser les modalités de cette distribution de semences. De nouvelles modalités efficaces ne paraissent pas évidentes à mettre en œuvre car les parts relatives du coton $\mathrm{Bt}$ et du coton conventionnel à placer dépendent notamment du facteur climatique et ne peuvent être déterminées parfaitement à l'avance. Pour éviter la conséquence

\footnotetext{
${ }^{8}$ A la rigueur à la fraction des parcelles refuge près, là où l'installation de ces parcelles est de mise.
} 
fâcheuse d'une hétérogénéité de la qualité du coton fibre, il faudrait que les variétés de coton Bt et de coton conventionnel soient isogéniques (ne se différenciant que sur le seul caractère $\mathrm{Bt})$.

Si cette réorganisation n'est pas mise en place, on peut anticiper que les paysans s'arrangeront par eux-mêmes, en catimini, induisant ainsi mélange variétal et hétérogénéité de la qualité du produit final $^{9}$. On peut alors craindre que les impacts négatifs pour les paysans et les filières concernées soient bien plus importants que les gains économiques espérés.

\section{Conclusion}

Dans le débat sur les OGM que d'aucuns trouvent futiles [40], omissions, demi mensonges ou demi vérités, extrapolations abusives, sont monnaies courantes. S'y glissent même quelques cas de corruption ${ }^{10}$ [41]. L'interrogation sur la rigueur et l'intégrité morale des scientifiques à vanter les mérites du coton Bt n'est pas sans fondement non plus [42], et cette interrogation peut être étendue aux journaux scientifiques se livrant à la course pour publier les résultats relatifs au succès des OGM.

L'analyse réalisée s'est concentrée sur le cas du coton Bt. Elle indique que, à part la confirmation d'un surcoût élevé des semences dans tous les pays utilisateurs, il y a fréquemment exagération sur les effets anticipés, qu'ils soient positifs ou négatifs.

L'utilisation du coton Bt n'affranchit pas des traitements chimiques et ne peut pas les affranchir totalement. Cette utilisation ne réduit pas forcément les coûts du contrôle des ravageurs. Les conditions d'utilisation, techniques et économiques, présentent un peu plus de diversité qu'initialement évoquée.

Les impacts observés du coton Bt diffèrent entre les pays et les modes de production. Dans les pays développés, l'utilisation de ce coton dans les grandes exploitations ne dégage pas un très net intérêt économique, mais le gain vient surtout du sentiment de 'confort' et de bonne conscience dans le contrôle des ravageurs.

Dans les PED, l'efficacité du coton Bt contre les ravageurs cibles est indéniable en permettant d'économiser 2-3 traitements chimiques. Le gain de rendement est aussi plus fréquent et plus net. Sur le plan technique, les perspectives de l'extension de l'utilisation de ce coton paraissent bonnes. Sur le plan économique, l'appréciation doit être plus nuancée. Il est rare de constater une réduction du coût total du contrôle des ravageurs parce que le surcoût des semences dépasse l'économie réalisée sur les insecticides. C'est le gain de rendement qui globalement permet de parvenir in fine à une rentabilité améliorée, au prix d'une perception accrue du risque financier. La sécurité économique dans l'utilisation du coton $\mathrm{Bt}$ dépend fondamentalement du coût des semences et des conditions économiques d'utilisation pour lesquels les pays disposent d'une marge de négociation, à condition de savoir tirer profit des acquis d'autres pays. En Inde, le prix des semences vient de baisser radicalement ${ }^{11}$, c'est fondamentalement le résultat d'une intervention des pouvoirs publics [43], rendue sans doute nécessaire par l'implication de la société civile. Il y a un espace de négociation sur le coût des semences de CGM, il serait regrettable que les pouvoirs publics des PED continuent à l'ignorer et à occulter le rôle qu'ils peuvent jouer.

\footnotetext{
${ }^{9}$ En Chine, la diffusion du CGM est responsable de multiplicité de variétés cultivées dans un même village, induisant un problème reconnu d'hétérogénéité de la qualité du coton produit.

${ }^{10}$ En 2005, Monsanto a été condamné en Indonésie pour corruption de fonctionnaire dans les procédures de biosécurité.

${ }^{11}$ Depuis juin 2006, le paquet de 450 g de semences hybrides de coton-Bt en Inde est passé 1650-1700Rs à 750Rs.
} 
Avec le recul de dix ans dans l'utilisation du coton Bt, on constate que les anticipations sur les effets ont été imparfaites, comme on vient de voir, et surtout très incomplètes. En quelques années, l'utilisation du coton Bt permet de ramener la pression parasitaire à un niveau plus faible et plus facile à gérer. C'est une évolution très positive qui doit conduire à cesser de raisonner l'utilisation du coton Bt suivant l'hypothèse d'un taux de couverture de 100\% (hors parcelles de refuge). Dans les PED, la co-habitation du CGM avec du coton conventionnel est à rechercher pour des raisons économiques et écologiques, mais elle suppose une réorganisation de la distribution des semences qui n'est pas évidente à mettre en oeuvre.

La focalisation sur la crainte de l'émergence de la résistance des ravageurs aux toxines Bt, non encore avérée, a occulté le possible changement d'équilibre entre les ravageurs de la culture cotonnière. Aujourd'hui, une chenille vorace tend à prendre la place de la principale chenille contrôlée par le coton Bt. L'utilisation du coton Bt ne peut être la solution définitive au problème du contrôle des ravageurs. Son application appelle à davantage de raisonnement pour être durable.

\section{Références bibliographiques}

1 Pinstrup-Andersen Per, etSchioler Ebbe Seeds of contention : world hunger and the global controversy over genetically modified crops, IFPRI, Washington D.C. Food Policy Statement 2001 2p.

2. ADB Agricultural biotechnology, poverty reduction and food security, Asian Development Bank, Manila (Philippines). Working Paper 2001 225p.

3. Prakash C.S. Global Hunger - Can Agricultural Biotechnology Help?, in, Feeding a Hungry World: The Moral Imperative of Biotechnology Rome, Italy.September 24, 2004.2004,pp. 7

4. MacDonald Nick, 2003. Genetically Modified organisms - the last thing the developing world needs. http://globalvision.org/library/6/561

5. $\quad$ Mazoyer Marcel, 2000. La moitié de la paysannerie mondiale n'est pas solvable pour les grands laboratoires. http://www.lemonde.fr/

6. Genes from engineered grass spread for miles, study finds, 2004.

7. Myers Dorothy GM cotton fails to impress, Pesticides News, 1999, 44, 6.

8. Conner Anthony J., Glare Travis R., etNap Jan-Peter The release of genetically modified crops into the environment. Part II. Overview of ecological risk assessment, The Plant Journal, 2003, 33, 19-46.

9. Kelemu Segenet, Mahuku George, GFregene Martin, et al. Harmonizing the agricultural biotechnology debate for the benefit of African farmers, 2003.

10. Gianessi Leonard P., Silvers Cressida S., Sankula Sujatha, etCarpenter Janet E. Plant biotechnology: Current and potential impact for improving pest management in U.S. agriculture. An analysis of 40 case studies. Insect Resistant cotton, NCFAP. 2002 20p.

11. Hofs J.L., Fok A.C. Michel, Gouse Marnus, etKirsten Johann Diffusion du CGM dans une filière instable en Afrique du Sud et les leçons pour l'Afrique Zone Franc, Revue Tiers Monde, 2006, à paraître.

12. Chaudhry Rafiq Impact of genetically engineered cotton in the world, in, Second Meeting of the Asian Cotton research and Development Network Tashkent, Uzbekistan.November 14-16, 2002.2002,pp. 4

13. Fok A.C. Michel, Liang WeiLi, Wang Guiyan, etWu Yuhong Diffusion du coton génétiquement modifié en Chine : leçons sur les facteurs et limites d'un succès, Economie Rurale, 2005, 285, 5-32.

14. Xu Jianxiang, You Zhibin, Wang Weiqin, etYang Yizhong Economic analysis of Bt cotton Planting in Jiangsu, Journal of Yangzhou University (Agricultural and Life Science Edition), 2004, 25, 3, 65-69.

15. Magaña J.E.M., Garcia J.G., Rodriguez A.J. O., etGarcia J.M. O. Comparative analysis of producing transgenic cotton varieties versus non-transgenic variety in Delicias, Chihuahua, Mexico, in, Beltwide Cotton Conferences.1999,pp. 255-256

16. Russell Derek, etDeguine Jean Philippe Durabilité de la culture des cotonniers transgéniques en Chine et en Inde, Cahier d'agriculture, 2006, 15, 3, 54-59.

17. Bennett R., Ismaël Y., etMorse S. Explaining contradictory evidence regarding impacts of genetically modified crops in developing countries. Varietal performance of transgenic cotton in India, Journal of Agricultural Science, 2005, 143, 35-41.

18. Laws Forrest, Monsanto to seek arbitration on licensing dispute. Delta and Pine Land says arbitration filing is 'without merit', in Deltafarm Press, May 20, 2004 pp. 2.

19. Anon., 2006. Monsanto to develop 20 new cotton seed hybrids in India. http://www.checkbiotech.org/root/index.cfm?fuseaction=news\&doc id=12229\&start=1\&control= 
207\&page_start=1\&page_nr=101\&pg=1 Année de consultation 2006. Date de consultation 20/02/2006

20. Monsanto Semente de soja Roundup Ready. A Opçao é so sua, Monsanto. 2005 12p.

21. Qaim Matin Bt cotton in India: Field trial results and economic projections, World Development, 2003, 30, 12, 2115-2127.

22. Orphal Jana Comparative analysis of the economics of Bt and Non-Bt cotton production, Institute of economics in Horticulture, Univ. of Hannover, Hannover, Germany. Pesticide Policy Project Publication Series. Special Issue 2005 71p.

23. Kerby Thomas A. What changes in yield and quality are due to transgenic varieties?, The cotton gin and oil mill press, 2001, 5-7.

24. Fitt Gary P., Wilson Lewis, Mensah Robert, etDaly Joanne Advances with integrated pest management as a component of sustainable agriculture: the case of the Australian cotton industry, in, 4th International Crop Science Congress Brisbane, Australia.26 Sept - 1 Oct. 2004.2004,pp. 11

25 M. Gouse, J. Kirsten, and L. Beyers, in Working Paper, 2002 p. 13 Department of Agricultural Economics, Extension and Rural development. University of Pretoria, Pretoria.

26. Fitt Gary P. Deployment and impact of transgenic Bt cotton in Australia, in (N.G., K., Ed.),The economic and environmental impacts of Agbiotech, New York, Kluwer,2003, 141-164

27 R. Bennett, Y. Ismaël, U. Kambhampati, and S. Morse, Economic impact of genetically modified cotton in India, AgBioForum 7 (2004) 96-100.

28 R. Devraj, A mindless conviction. Despite failures and warnings, GM foods find a supportive government, India Together. http://www.indiatogether.org/agriculture/articles/gmanyway.html Access on $06 / 04 / 2006$

29. Anon., Bt cotton co invites AP govt's ire, in The Economic Times Online, 01/09/2006 pp. 1.

30. Hofs Jean Luc, Fok A.C. Michel, et Vaissayre Maurice Impact of Bt cotton adoption on pesticide use by smallholders: A 2-year survey in Makhatini Flats (South Africa), Crop Protection, 2006, in press.

31. Huang Jikun, Hu Ruifa, Pray Carl E., Qiao Fangbin, etRozelle Scott Biotechnology as an alternative to chemical pesticides: a case study of Bt cotton in China, Agricultural Economics, 2003, 29, 1, 55-67.

32 S. Lang, Seven-Year glitch: Cornell warns that Chinese GM cotton farmers are losing money due to secondary pests. http://www.news.cornell.edu/stories/July06/Bt.cotton.China.ssl.html Access on 01/08/2006

33. Bennett Richard, Ismaël Youssouf, etMorse Stephen Reductions in insecticide use from adoption of Bt cotton in South Africa: impacts on economic performance and toxic load to the environment, Journal of Agricultural Science, 2004, 142, 665-674.

34. Thirtle Colin, etJenkins Beyers Lindie Can GM-technologies help African smallholders? The impact of Bt cotton in the Makhatini Flats of Kwazulu-Natal, World development, 2003, 31, 4, 717-732.

35. ISAAA, 2005. Bt cotton: Crop losses due to insect pests of cotton and the costs of control. http://www.isaaa.org/kc/Global\%20Status/crop/gmcotton/croplosses.htm Année de consultation 2006. Date de consultation 02/08/2006

36. Qaim Matin, etde Janvry Alain Genetically modified crops, corporate pricing strategies, and Farmers' adoption: the case of Bt cotton in Argentina, Amer. J. Agr. Econ., 2003, 85, 4, 814-828.

37. Trigo Eduardo, Chudnovsky Daniel, Cap Eugenio, etLópez Andrés Los transgénicos en la agricultura argentina. Una historia con final abierto, International Institute for Sustainable Development, Winnipeg (Canada). Trade Knowledge Network Paper 2003 109p.

38. Pray Carl, Bengali Prajakta, etRamaswami Bharat The cost of biosafety regulations: the Indian experience, 2005.

39. Li Fangdeng Prodenia litura (Fabricius): factors of its outbreak and how to control it, Anhui Agriculture, 2004, 7, 20.

40. Elliesen Tillmann Gain in Indian cotton yield? The futile dispute over genetic engineering, $D+C$, 2003, 30, 2003.5, 207-209.

41. Gala Rhea, GM Cotton: corruption, hype, half-truths and lies, in Institute of Science in Society Press Release, 21/01/2005 pp.

42. Rao Kameswara C., 2003. The ballgame of Bt cotton in India. http://www.fbae.org/Channels/agri_biotech/cotton/ballgame_of_bt... Année de consultation 2006. Date de consultation 24/02/2006 
